\title{
Effect of consumption of soy isoflavones on behavioural, somatic and affective symptoms in women with premenstrual syndrome
}

\author{
M. Bryant ${ }^{1}$, A. Cassidy ${ }^{2}$, C. Hill ${ }^{1}$, Jonathan Powell ${ }^{3}$, Duncan Talbot ${ }^{3}$ and L. Dye ${ }^{1 *}$ \\ ${ }^{1}$ BioPsychology Group, Institute of Psychological Sciences, University of Leeds, Leeds LS2 9JT, UK \\ ${ }^{2}$ School of Medicine, Health Policy \& Practice, University of East Anglia, Norwich NR4 755, UK \\ ${ }^{3}$ Unilever R\&D, Colworth, Sharnbrook, Bedfordshire MK44 1LQ, UK
}

(Received 18 June 2004 - Revised 4 December 2004 - Accepted 13 December 2004)

\begin{abstract}
Up to $80 \%$ of the Western female population experience premenstrual syndrome (PMS). Long-term pharmacological therapy is unacceptable to most women, and is not warranted for moderate symptoms. Nutritional therapies are popular, but lack a clear evidence base. Anecdotal evidence suggests beneficial effects of soy isoflavones because of their influence on endogenous oestrogen and actions on specific tissues. The effect of isolated soya protein (ISP) containing $68 \mathrm{mg} / \mathrm{d}$ (aglycone equivalents) soy isoflavones (IF) on premenstrual symptom severity was studied in a seven-menstrual cycle, double-blind, placebo-controlled, crossover intervention study in twenty-three women with prospectively confirmed PMS aged $18-35$ years and BMI $19-30 \mathrm{~kg} / \mathrm{m}^{2}$. ISP containing IF or milk protein placebo was consumed for two complete menstrual cycles. ISP containing IF (genistein, daidzein, equol) were measured in $24 \mathrm{~h}$ urine samples. After two cycles of ISP containing IF intervention, total symptoms $\left(F_{(2,36)} 8 \cdot 20, P=0 \cdot 000\right)$ and physical symptoms $\left(F_{(2,36)} 8 \cdot 18, P=0 \cdot 000\right)$ were significantly reduced compared with baseline after both active and placebo treatments, although differences between active and placebo treatment were non-significant. Specific premenstrual symptoms, headache $\left(F_{(2,32)} 4 \cdot 10, P=0.026\right)$ and breast tenderness $\left(F_{(2,32)} 4 \cdot 59, P=0 \cdot 018\right)$, were reduced from baseline after soy IF, but not milk protein placebo. Cramps $\left(F_{(2,32)} 4 \cdot 15, P=0 \cdot 025\right)$ and swelling $\left(F_{(2,32)} 4 \cdot 64, P=0 \cdot 017\right)$ were significantly lower after active treatment compared with placebo. Concentrations of genistein and daidzein were increased following soy IF consumption, but equol production did not enhance symptom reduction. The present study showed that ISP containing IF may have potential to reduce specific premenstrual symptoms via non-classical actions.
\end{abstract}

Phyto-oestrogen: PMS: Nutrient: Menstrual: Hormone: Soy

Premenstrual syndrome (PMS) is characterised by a collection of behavioural, somatic and affective symptoms, which occur in the $7-10 \mathrm{~d}$ prior to the onset of menstruation and are relieved at or shortly after commencement of menstrual flow. Prevalence is common, with between 30 and $80 \%$ of Western premenopausal women reporting premenstrual symptoms (Singh et al. 1998). Although more than 200 symptoms have been associated with PMS, common symptoms that classically characterise the syndrome include depression, irritability, mood swings, water retention-based symptoms (e.g. breast tenderness and bloating), changes in appetite and food cravings (Freeman, 2003).

The aetiology of PMS is still not clearly defined (for review, see Halbreich, 2003). Symptoms have been attributed to ovarian hormones, but there is little convincing evidence of biochemical differences between women with and without PMS (Rubinow, 1992). The current consensus suggests that PMS is a psychoneuroendocrine phenomenon. Normal ovarian function, rather than hormone imbalance, acts as a trigger operating via the hypothalamic-pituitary-gonadol axis to influence neurotransmitter function in the central nervous system (Reid \& Yen, 1981; Ashby et al. 1988; Rubinow \& Schmidt, 1995; Walker, 1995; Steiner et al. 1997; Korzekwa \& Steiner, 1999). Ovarian suppression with agonists of gonadotrophin-releasing hormone has been shown to reduce symptoms (Muse et al. 1984). It is therefore possible that PMS is triggered by hormone-related events. Psychological disturbances account for many symptoms of PMS, suggesting that peptides originating from the brain may be important mediators of symptoms. Hence, the serotonergic system is implicated in the aetiology of PMS. Long-term acceptability of pharmacological treatment is low, and for many women, symptoms are not severe enough to warrant long-term use of drug therapy. Hence, alternative therapies, such as supplementation with specific nutrients, are often sought by women. A number of nutrients have been recommended, including $\mathrm{Ca}, \mathrm{Mg}$, pyridoxine and $\gamma$-linolenic acid. Overall, the quality of research examining the efficacy of such nutrients is questionable (e.g. inadequate participant numbers, poor or absent control groups, failure to correctly identify the premenstrual phase or adequately diagnose PMS) and the biological mechanisms of many nutrients have not been elucidated (for a review, see Bryant \& Dye, 2004). Consumption of phyto-oestrogens by women has been proposed to have beneficial effects by the popular press, but evidence is anecdotal.

Phyto-oestrogens may be effective at reducing some premenstrual symptoms because they have the ability to act as antioxidants, inhibit angiogenesis, facilitate neurobehavioural actions, and exhibit weak oestrogenic and anti-oestrogenic effects (Cassidy et al. 1994; Setchell \& Cassidy, 1999; Lephart et al. 2001, 
2003). It is therefore plausible that phyto-oestrogens may reduce premenstrual symptoms by stabilising the natural cyclical fluctuation of oestrogen. However, it is important to acknowledge that the literature on the hormonal effects of isoflavones (IF) is not conclusive and there are some discrepancies in the literature (e.g. relating to optimal dosage of IF). Furthermore, some studies have shown an effect on hormones of soy intervention with IF removed (for a review, see Hill \& Dye, 2003).

In man, there are large inter-individual variations in the metabolism of IF, especially in the production of equol from daidzein (Rowland et al. 2003). Equol is more oestrogenic than daidzein and is therefore believed to exert greater biological actions (Brienholt \& Larsen, 1998). Recent data suggest that the mechanism of action and effectiveness of soy in hormone-dependent studies is largely dependent upon equol production (Setchell et al. 2002). Only about $30 \%$ of adults are able to produce equol (Setchell et al. 1984; Rowland et al. 2000). Thus, the clinical effectiveness of IF may relate to the ability to produce equol and changes in symptoms after consumption may well relate to individual variation in equol production.

Although IF have been shown to reduce cyclical mastalgia and menstrual migraines in a small number of studies (McFadyen et al. 2000; Burke et al. 2002; Ingram et al. 2002), which is indicative of both a central and peripheral action of IF, to date there have been few clinical trials investigating the effectiveness of IF for PMS. There has been only one study, to date, which has examined the effect of soy IF on global symptoms of PMS. Preliminary findings from this study suggest that $40 \mathrm{mg} / \mathrm{d}$ soy IF can reduce premenstrual headaches and physical symptoms in women with PMS (Ishiwata et al. 2003).

The primary aim of the present study was to determine whether isolated soya protein (ISP) containing IF were more beneficial than placebo in relieving symptoms of PMS in a seven-cycle randomised double-blind placebo-controlled crossover trial and to assess whether urinary excretion of phytoestrogens was related to the endpoint measures. The effectiveness of ISP containing IF was assessed by comparing symptom severities during the menstrual cycle in women consuming ISP containing $68 \mathrm{mg} / \mathrm{d}$ (aglycone weight) soy IF with symptomatology during two baseline and two placebo-treated cycles. Total symptom severity and severity of individual symptoms were examined in order to determine specificity of effects. ISP containing IF were also measured in urine to gauge compliance (genistein and daidzein) and to determine potential relationships between symptom change and equol production.

\section{Methods}

\section{Experimental design}

The study conformed to a double-blind, placebo-controlled, crossover design with two conditions of ISP containing $68 \mathrm{mg} / \mathrm{d}$ soy IF or an identical placebo product. Participants were assigned an identity number chronologically, based on when they volunteered to take part in the research. Twelve participants consumed the active soy IF and eleven consumed milk protein placebo during the first intervention period before crossover and the doubleblind nature of the study was maintained until the end of the study.

Active constituents. Food supplements consisted of a powder that could be made into a drink (or sprinkled over food) and a snack bar. One serving of powder and one bar were consumed each day, providing a total of $30.5 \mathrm{~g}$ ISP, containing $68 \mathrm{mg} / \mathrm{d}$
IF (aglycone equivalents; Solae ${ }^{\mathrm{TM}}$ ). These were formulated and produced to good manufacturing practice by Solae ${ }^{\mathrm{TM}}$ to ensure standardised reproducible doses of ISP containing IF to guarantee quality control.

Placebo constituents. Placebo products were identical in appearance and taste to the active products, but contained milk protein instead of ISP. Both products were coded numerically to maintain blinding.

Product consumption. After two cycles of screening, participants were instructed to consume the products as part of their habitual diet for two menstrual cycles (active or placebo). In doing so, there was the potential for energy intake to increase by approximately $300 \mathrm{kcal} / \mathrm{d}$ in both the active and placebo groups. However, to avoid weight gain, participants were advised on how to incorporate the products into their diet. The first intervention phase (two menstrual cycles) was followed by one cycle of washout and then a further two cycles of either active treatment or placebo after crossover.

\section{Outline of study protocol}

Participants. Participants were recruited from within the local area by posters, fliers and email distribution advertisements. Inclusion criteria were: (1) presence of regular menstrual periods; (2) not using any hormonal preparations; (3) not taking over-thecounter preparations for PMS or any other condition; (4) not following a weight loss diet; (5) not suffering from depression other than premenstrually; (6) no intolerance, hypersensitivity or dislike to foods containing soya; (7) not pregnant, lactating or planning pregnancy; (8) no known diabetic condition or acute conditions of hepatic, renal, cardio, pulmonary, digestive, haematological, neurological or thyroidal disease. In total, 142 women volunteered to take part in the study. Fifty-one women aged 18-35 years with a BMI $19-30 \mathrm{~kg} / \mathrm{m}^{2}$ met the inclusion and exclusion criteria. Forty-one began two screening cycles and twenty-three went on to complete the seven-cycle intervention. All but one of the participants who dropped out stopped during the twocycle screening period. The main reasons for dropout were poor taste acceptability of the food products (participants were encouraged to try products before the intervention) and burden. Dropouts were experienced early in the study, so that intention to treat analysis could not be performed, since this was a post-randomisation loss and no data on the primary endpoints were obtained after loss to follow up. Here, we present data from twenty-three women who completed the entire study. Written consent was obtained from each individual and the study was approved by the ethics committee of the School of Psychology (University of Leeds)

Table 1. Sample characteristics

(Mean values with their standard deviations)

\begin{tabular}{|c|c|c|c|c|c|c|}
\hline \multirow[b]{2}{*}{ Characteristic } & \multicolumn{2}{|c|}{ Total $(n 23)$} & \multicolumn{2}{|c|}{$\begin{array}{l}\text { Active, first } \\
\text { cycle (n 12) }\end{array}$} & \multicolumn{2}{|c|}{$\begin{array}{l}\text { Placebo, first } \\
\text { cycle }(n 11)\end{array}$} \\
\hline & Mean & SD & Mean & SD & Mean & SD \\
\hline Age (years) & $33 \cdot 65$ & 5.85 & $32 \cdot 25$ & $5 \cdot 28$ & $35 \cdot 18$ & $6 \cdot 31$ \\
\hline BMI $\left(\mathrm{kg} / \mathrm{m}^{2}\right)$ & $23 \cdot 28$ & $3 \cdot 14$ & $23 \cdot 78$ & 3.54 & $22 \cdot 79$ & $2 \cdot 77$ \\
\hline Subjective health $(1-6)$ & $4 \cdot 78$ & 1.04 & 4.58 & $1 \cdot 16$ & $5 \cdot 0$ & 0.89 \\
\hline Dietary restraint* ${ }^{\star}$ & $2 \cdot 37$ & $2 \cdot 13$ & $2 \cdot 39$ & 0.79 & $2 \cdot 33$ & 0.94 \\
\hline
\end{tabular}

* Scores calculated from standard procedures for Dutch Eating Behaviour Questionnaire (VanStrien et al. 1986). 
and the United Leeds Teaching Hospitals Ethics Committee. Participant characteristics are shown in Table 1 according to treatment order. Age, BMI, subjective health and dietary restraint were similar between women taking placebo first compared with those taking active treatment first.

Symptom measurements. There are no guidelines for quantifying the degree of severity or frequency of PMS (Freeman, 2003). Nor is there a consensus for the diagnosis of clinically significant PMS. Thus, diagnosis should be based on marked fluctuations in symptoms. Because PMS is defined by the presence, severity and timing of symptoms, each of these factors should be considered (Schnurr, 1989). For periods other than the premenstrual phase, symptoms should be sporadic and no different to those experienced by the rest of the population (Mortola, 1992). Symptoms that are distressing must occur premenstrually and remit with menses. It is therefore essential that prospective measures are employed during the diagnosis and testing of intervention studies. In the present study, symptoms were measured daily from the first day of menstruation in cycle one through to the first day of menstruation in cycle eight using the Penn Daily Symptoms Rating (DSR; Freeman et al. 1996). This is a validated, single-page, self-report questionnaire in which seventeen symptoms are rated on a five-point Likert scale $(0=$ not present at all, $4=$ severe $)$. Freeman et al. (1996) grouped symptoms into the sub-clusters of: mood (irritability, mood, tension, anxiety, depression, loss of control), behaviour (poor coordination, confusion, insomnia, crying, fatigue, headaches), pain (breast tenderness, cramps, aches) and physical symptoms (food cravings, swelling). Analysis of symptoms in the present study considers these clusters, as well as the individual symptoms that comprise them. Participants were asked to rate the presence and severity of these commonly reported premenstrual symptoms every evening in the DSR, which was provided in the form of a daily diary covering 1 week. Diaries were returned weekly using freepost envelopes to prevent the identification of patterns of symptoms and reduce the likelihood of demand characteristics producing stereotypical patterns of reporting. This measure was used to diagnose PMS and assess symptom severity (during the intervention). In order for participants to be eligible, they were required to demonstrate an increase in overall symptoms that was at least $30 \%$ greater premenstrually $(6 \mathrm{~d}$ before menstruation) than during days 5-10 of the menstrual cycle (after menstruation) for at least one of two screening months based on recommendations from the National Institute of Mental Health (1983) workshop.

Metabolism of soy isoflavones. Each participant collected four $24 \mathrm{~h}$ urine samples during the premenstrual phase of each intervention treatment cycle (two during active and two during placebo) to examine individual differences in metabolism following the intervention, and as a measure of compliance to the dietary protocol. Genistein $(\mathrm{mg} / \mathrm{d})$, daidzein $(\mathrm{mg} / \mathrm{d})$ and equol $(\mathrm{mg} / \mathrm{d})$ were measured in the samples using monoclonal antibody-based Auto dissociation enhanced lanthanide fluoro immunoassay) (Delfia) assay technology. Delfia assays are commonly ten times more sensitive than conventional enzyme immunoassays. The monoclonal antibodies to genistein and equol were the property of Unilever and the daidzein monoclonal was obtained from Fortune Kohen, Weizmann Institute. The laboratory implemented its own internal quality control procedures by determination of assay coefficients of variance. Plates were repeated if they did not meet the low, medium and high quality control pass/fail criteria. Individual samples were repeated if the coefficient of precision was about $10 \%$. All of the assays were run in duplicates and averaged. Assays were validated against published BC-MS (Adlercreutz, Helsinki) and commercial (Labmaster) polyclonal Delfia methods.

\section{Statistical analysis}

Data were analysed using the SPSS for Windows (version 11; SPSS Inc., Chicago, IL, USA) statistical software package.

The effect of the intervention was examined using $3 \times 2 \times 4$ doubly multivariate ANOVA (MANOVA). The MANOVA comprised three factors: treatment with three levels (baseline, soy IF and placebo); cycle with two levels (first and second cycle of each treatment); cycle phase with four levels (during menstruation, after menstruation, rest of cycle and premenstrual). Analysis was performed separately for total symptoms and for each group of symptoms that comprised a symptom cluster (i.e. five tests: total; mood; behaviour; pain; physical). This technique is appropriate for the analysis of repeated measures designs, which include multiple dependent variables. It protects against elevated family-wise error rates associated with repeated univariate testing (Tabachnick \& Fidell, 1996). A significant multivariate $F$ for the symptom cluster is required in order to consider the effects of intervention on individual symptoms that comprised that cluster. However, because this is an exploratory study and because the likelihood of systematic effects producing a significant multivariate $F$ is dependent on the robustness of the factor structure of the DSR, significant univariate results for specific symptoms are also cautiously described. Post hoc comparisons using Bonferroni correction that employed to examine significant effects of treatment, cycle, phase or interactions of these independent variables.

Further statistical analysis considered the second cycle of intervention only. Placebo effects can be strong in the first cycle of intervention and quite resistant in PMS (Freeman \& Rickels, 1999) but should begin to decline to baseline as the study continues. Furthermore, repeated exposure to soy may be required to reach steady concentrations or affect receptor sensitivity, although to date, evidence for the time to affect cyclical symptoms is not available. This study comprised two cycles of intervention, and it was hypothesised that effects should be greater in the second cycle for these reasons. Therefore, separate MANOVA were performed, examining total symptoms and symptom clusters in the second cycle only. Each had a between-subjects factor of 'treatment' (baseline, second cycle soy IF, second cycle placebo).

Pearson's product moment correlation coefficients were performed to examine the relationship between initial premenstrual symptom severity and symptom change after treatment for placebo and active interventions. Differences between the correlation coefficients for active and placebo treatments were tested using Fisher- $r$ tests to examine the difference between two independent correlation coefficients, based on procedures outlined by Cohen $\&$ Cohen (1983).

Doubly MANOVA were conducted to examine the effect of intervention on the level of genistein and daidzein, with 'treatment' as a within-subjects factor (active/placebo). For equol, a MANOVA was performed to investigate whether equol and non-equol producers differ in their reported change in symptom 
Table 2. Baseline daily symptom scores

(Mean values with their standard deviations)

\begin{tabular}{|c|c|c|c|c|c|c|}
\hline \multirow[b]{2}{*}{ Cycle phase } & \multicolumn{2}{|c|}{ Total (n 23) } & \multicolumn{2}{|c|}{$\begin{array}{l}\text { Active, first } \\
\text { cycle }(n 12)\end{array}$} & \multicolumn{2}{|c|}{$\begin{array}{l}\text { Placebo, first } \\
\text { cycle }(n 11)\end{array}$} \\
\hline & Mean & SD & Mean & SD & Mean & SD \\
\hline During menstruation† & $12 \cdot 30$ & $7 \cdot 86$ & $15 \cdot 31$ & $7 \cdot 83$ & $9 \cdot 03$ & $6 \cdot 77$ \\
\hline After menstruation $†$ & $5 \cdot 46$ & 5.06 & $7 \cdot 92$ & $5 \cdot 60$ & $2 \cdot 76^{\star}$ & $2 \cdot 56$ \\
\hline Rest of cycle & $6 \cdot 13$ & $5 \cdot 70$ & 7.93 & $6 \cdot 08$ & $4 \cdot 15$ & $4 \cdot 75$ \\
\hline Premenstrual† & 13.40 & $9 \cdot 70$ & $16 \cdot 52$ & 11.51 & $10 \cdot 00$ & 6.07 \\
\hline
\end{tabular}

Mean values were significantly different between treatment orders, ${ }^{*} P<0.05$.

† Mean values for $6 \mathrm{~d}$ during, after and premenstrual phases.

$\ddagger$ Calculation of mean value depends on individual cycle length (i.e. from days 11 to -7 of the menstrual cycle).

severity after intervention. Equol production was therefore included as a between-subjects factor.

\section{Results}

\section{Compliance}

Excretion of genistein and daidzein increased following active treatment but not placebo (daidzein: $F_{(1,7)} 43 \cdot 05, P=0 \cdot 001$; genistein: $\left.F_{(1,7)} 26 \cdot 4, P=0.002\right)$, indicative of adherence to the regimen, although a high degree of inter-individual variation was observed (Table 5; see p. 735).

\section{Baseline symptoms}

Mean total severity baseline scores during four phases of the menstrual cycle are shown in Table 2 according to the order in which active or placebo treatments were administered. Baseline scores did not differ according to treatment order, except for the phase after menstruation, when those receiving placebo first had lower symptoms $\left(F_{(2,36)} 7 \cdot 8, P<0 \cdot 05\right)$. Significant cycle phase effects were observed $\left(F_{(3,66)} 19.22, P<0.001\right)$ such that cyclicity was demonstrated, with symptoms reported to be most severe during the premenstrual phase of the cycle.

\section{Effect of intervention on total symptoms}

Mean premenstrual total symptom scores for the first and second cycles of active and placebo interventions are shown in Table 3. Total symptoms were reduced compared with baseline following both active and placebo treatments (multivariate $F_{(10,50)} 2 \cdot 45$,
$P=0 \cdot 018$ ). Reductions (change from baseline) in symptoms are shown in Fig. 1 according to menstrual cycle phase. Overall symptom reduction was greater following active treatment compared with placebo during the premenstrual phase and a significant treatment by phase interaction was observed (multivariate $\left.F_{(6,84)} 2.75, P=0.017\right)$. However, main effects between treatment were non-significant. Observed power for these effects (treatment, phase and treatment by phase interaction) were 0.99, 1.00 and $1 \cdot 00$, respectively (i.e. sufficient post hoc power was demonstrated). When treatment order was included as a between-subjects factor, it had no effect on change in premenstrual symptoms between baseline and intervention (multivariate $F_{(2,20)} 0 \cdot 72$, NS).

\section{Effect of treatment on symptoms in the second cycle of intervention}

The main effect of treatment on premenstrual symptomatology in the second cycle of intervention was highly significant (multivariate $\left.F_{(10,66)} 5 \cdot 52, \quad P=0 \cdot 0010\right)$. Step-down univariate analysis revealed significant reductions from baseline in symptoms after both active and placebo interventions for total premenstrual symptoms (multivariate $F_{(2,36)} 8 \cdot 20, P=0 \cdot 000$ ) and premenstrual symptoms in each of the symptom clusters; mood (multivariate $F_{(2,36)} 15.91, P=0.000$ ), behaviour (multivariate $F_{(2,36)} 24.48$ $P<0 \cdot 01$ ), pain (multivariate $F_{(2,36)} 16 \cdot 11, P=0 \cdot 000$ ) and physical symptoms (multivariate $F_{(2,36)} 8 \cdot 18, P=0 \cdot 000$ ). Pairwise comparisons revealed that significant reductions in premenstrual symptoms occurred between baseline and soy IF treatment for total symptoms and all symptom clusters. However, placebo treatment reduced reports on the symptom clusters mood, behaviour and pain, but not total symptoms or physical symptoms. The difference in symptom scores between active and placebo treatments was non-significant for total symptoms and all symptom clusters, although significance was approached in the physical symptoms cluster (Fig. 2).

Effect of intervention on individual premenstrual symptoms in the second treatment cycle

Main effects of treatment were observed for headache $\left(F_{(2,32)}\right.$ $4 \cdot 10, P=0 \cdot 026)$ and breast tenderness $\left(F_{(2,32)} 4 \cdot 59, P=0 \cdot 018\right)$. These were significantly reduced from baseline after active treatment, but not after placebo. However, the differences between active and placebo treatments were non-significant. Conversely, there was a significant main effect of treatment on cramps

Table 3. Change in mean premenstrual scores after active and placebo interventions

(Mean values with their standard deviations)

\begin{tabular}{|c|c|c|c|c|c|c|c|c|c|c|}
\hline \multirow[b]{3}{*}{ Symptom cluster } & & & \multicolumn{4}{|c|}{ Active } & \multicolumn{4}{|c|}{ Placebo } \\
\hline & \multicolumn{2}{|c|}{ Baseline } & \multicolumn{2}{|c|}{ First cycle } & \multicolumn{2}{|c|}{ Second cycle } & \multicolumn{2}{|c|}{ First cycle } & \multicolumn{2}{|c|}{ Second cycle } \\
\hline & Mean & SD & Mean & SD & Mean & SD & Mean & SD & Mean & SD \\
\hline Total & 13.91 & $9 \cdot 70$ & $9 \cdot 67$ & 8.40 & 9.35 & 9.43 & $9 \cdot 14^{*}$ & 7.92 & 11.41 & $10 \cdot 75$ \\
\hline Mood & $7 \cdot 68$ & 6.43 & $3.03^{\star \star \star}$ & 3.41 & $3.65^{\star *}$ & 4.49 & $2 \cdot 35^{\star \star \star}$ & $2 \cdot 84$ & $4 \cdot 06^{*}$ & 4.52 \\
\hline Behavioural & 7.00 & 5.02 & $3.56^{\star}$ & 3.70 & $3 \cdot 28^{\star \star \star}$ & $3 \cdot 12$ & $3.43^{\star \star}$ & 2.98 & $3.54^{\star \star \star}$ & 3.43 \\
\hline Pain & 3.35 & 2.78 & $1 \cdot 37$ & $1 \cdot 64^{\star \star \star}$ & $1.51^{\star \star *}$ & 1.68 & $1.90^{\star \star \star}$ & $1 \cdot 87$ & $1.99^{\star \star \star}$ & 2.03 \\
\hline Physical & 2.49 & 2.00 & $1 \cdot 38$ & $1 \cdot 18^{*}$ & $1.08^{\star \star \star}$ & $1 \cdot 16$ & $1.46^{\star *}$ & 1.37 & $1 \cdot 82$ & 1.58 \\
\hline
\end{tabular}

${ }^{\star} P<0.01 ;{ }^{\star \star} P<0.001 ;{ }^{\star \star \star} P<0.0001$ (significant reduction from baseline). 
(A)

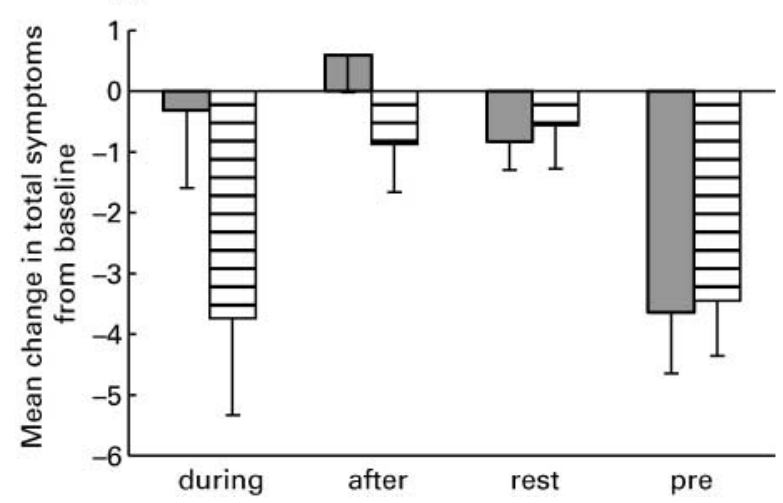

(B)

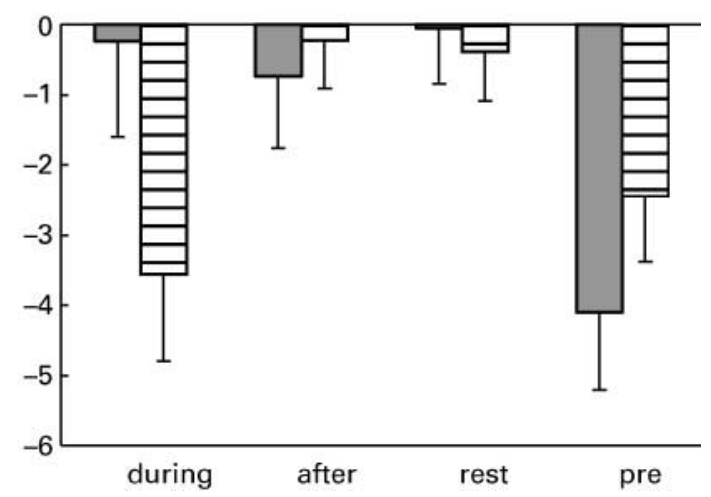

Fig. 1. Comparison of symptom change after baseline between first (A) and second (B) cycles of intervention. Values are means with their standard errors shown by vertical bars. Menstrual cycle phases: during, during menstruation; after, after menstruation; rest, rest of cycle; pre, premenstrual. $\square$, Isolated soya proteins with isoflavones; 目, milk protein placebo.

$\left(F_{(2,32)} 4 \cdot 15, P=0 \cdot 025\right)$ and swelling $\left(F_{(2,32)} 4 \cdot 64, P=0 \cdot 017\right)$, which improved more after active treatment compared with placebo, although reductions from baseline were non-significant. There were no effects of treatment on any other individual symptoms.

Relationship between initial premenstrual symptom severity and symptom change after treatment for placebo and active interventions

A significant positive correlation between mean premenstrual symptom score at baseline and symptom change from baseline to active treatment was found for total premenstrual symptoms $(r$ $4.45, n 23, P=0.032)$ but not for placebo treatment $(r 0.17, n 23$, $P=0.432$ ). Results for physical symptoms were similar; a significant positive correlation was only observed between baseline symptomatology and change in physical symptoms after active treatment ( $r 0.56, n 23, P=0.005)$. Thus, a higher initial severity of total and physical premenstrual symptoms was found to relate to a greater reduction in these symptoms after active treatment. Change in score on the mood, behaviour and pain symptom clusters was significantly related to mean baseline premenstrual symptoms, following both active and placebo treatments (Table 4).

The strength of the correlations appears greater following active treatment compared with placebo. However, Fisher- $r$ tests revealed no significant differences between the correlation coefficients for active and placebo treatments for total symptoms or any symptom clusters. Thus, the strength of the relationships between initial premenstrual symptom severity and change in

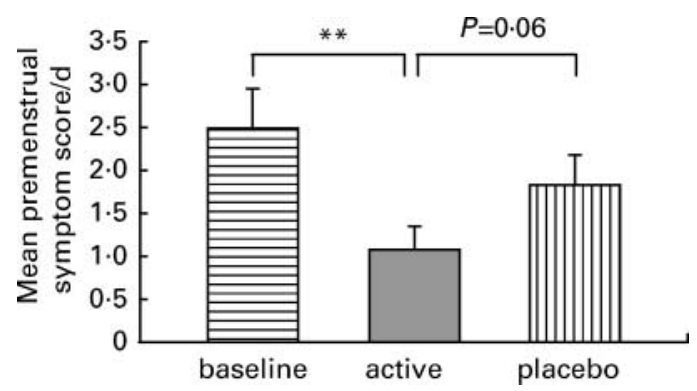

Fig. 2. Effect of treatment on physical premenstrual symptoms (second cycle). ${ }^{\star \star} P<0.01$. symptoms after treatment did not differ for active and placebo treatments.

\section{Influence of intervention on menstrual cycle length}

Cycle length was significantly increased between baseline and active, but not baseline and placebo treatment (Fig. $3 ; F_{(2,38)}$ $3.75, P<0.05)$.

\section{Metabolism of isolated soya proteins with isoflavones}

In total, six of the twenty-three participants $(26 \%)$ were equol producers (Table 6). Symptom reports did not differ between equol and non-equol producers for any symptom cluster (multivariate $F_{(18,1)} 0.58, P=0.793$ ). However, there was a trend for a greater reduction in total and physical symptoms in the equol producers compared with the non-equol producers during the premenstrual phase of the cycle.

\section{Discussion}

Consumption of ISP containing $68 \mathrm{mg}$ soy IF (aglycone equivalents) resulted in reductions to some premenstrual symptoms and altered menstrual cycle length. However, reductions in most symptoms relative to baseline were observed after both active and placebo treatments. Thus, the effect of placebo in the present 2-month treatment intervention study remained strong. Physical symptoms seemed to be most responsive to ISP in this small sample. Here, the difference between active and placebo treatments approached significance. Headache and breast tenderness

Table 4. Pearson's product moment correlation coefficients: relationship between premenstrual symptom severity at screening and premenstrual symptom change

\begin{tabular}{lcc}
\hline Symptom cluster & Active $(n$ 23) & Placebo $(n$ 23) \\
\hline Total & $0.49^{\star}$ & 0.17 \\
Mood & $0.70^{\star}$ & $0.57^{\star \star}$ \\
Behaviour & $0.63^{\star \star \star}$ & $0.66^{\star \star \star}$ \\
Pain & $0.63^{\star \star *}$ & $0.4^{\star \star \star}$ \\
Physical & $0.56^{\star \star}$ & 0.41 \\
\hline
\end{tabular}

${ }^{\star} P<0.05 ;{ }^{\star \star} P<0.01 ;{ }^{* \star \star} P<0.001$ 


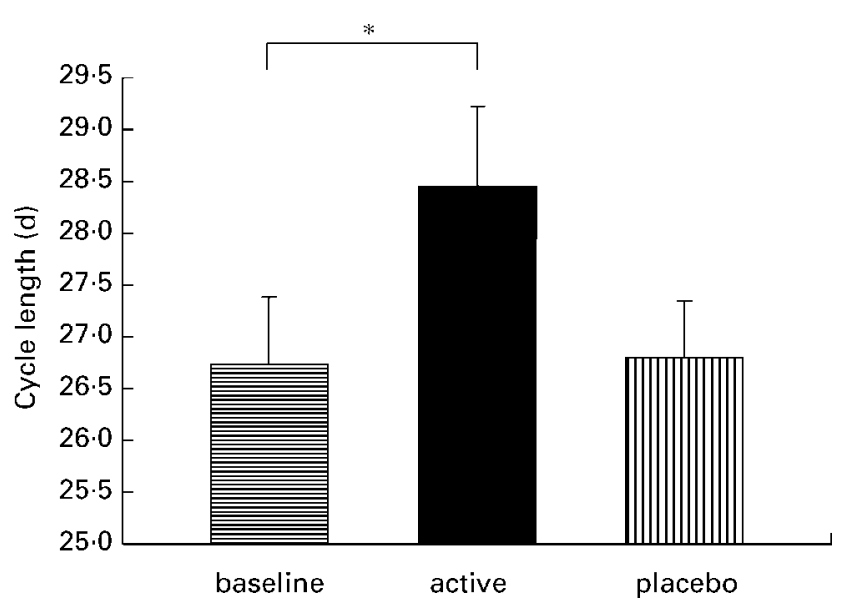

Fig. 3. Effect of soy isoflavones on menstrual cycle length. ${ }^{\star} P<0.05$.

symptoms were significantly reduced from baseline after active treatment, but not placebo. However, the interpretation of this effect must be tempered against the enduring placebo effects observed. Thus, in order for any specific treatment to be considered effective, benefits should be clearly demonstrated and significantly different from a placebo response. This was observed in the present study in terms of individual symptoms of cramps and swelling only. These symptoms are less vulnerable to psychological influences. Somatic symptoms are more resistant to placebo effects than mood and behavioural symptoms, which are more likely to be affected by subjective expectations (Freeman, 2003).

Comparisons of the change in symptoms observed in the present study with other PMS treatment studies are difficult because of the variety of tools that are used to assess PMS and the often retrospective reporting procedure. The scores themselves pertain to the measure used, due to considerable differences in the nature and number of symptoms in instruments (Freeman, 2003). Moreover, there are no widely accepted biological markers of PMS, so responses to treatment must be based on subjective reports. Coupled with this, there is high variation in symptom reports and these are not necessarily comparable between women. Furthermore, non-significant reductions in symptoms may not necessarily imply that they are not clinically important. Even small improvements may be clinically relevant to individuals.

The benefits of daily ISP containing IF consumption on physical symptoms have been reported previously (McFadyen et al. 2000; Ingram et al. 2002). The present study extends these findings by demonstrating that initial severity of symptoms is a mediating factor, such that women whose initial symptom severity is high experience a greater reduction in symptoms. This could be a statistical effect such as regression to the mean, although this is unlikely since the effect should be similar for both active and placebo responses and should interact with order of treatment. Alternatively, there is greater potential for change if initial symptom severity is high and lower probability of floor or ceiling effects. The similarity between findings of the present study and those of Ishiwata et al. (2003) are striking, in that both demonstrate a clear reduction primarily in symptoms of a somatic nature. This consistency between findings suggests a robust effect of ISP containing IF on somatic symptoms.

Placebo effects are common in psychosomatic conditions. PMS has been suggested to be particularly vulnerable to placebo effects, where most responses observed in the first cycle of intervention are created by a desire to improve symptoms felt by participants who believe that what they are taking is having an effect (Freeman \& Rickels, 1999). Thus, the magnitude of the placebo

Table 5. Urinary soy isoflavones after active and placebo treatments

(Mean values with their standard deviations, $n 20 \dagger$ )

\begin{tabular}{|c|c|c|c|c|c|c|c|c|}
\hline & \multicolumn{4}{|c|}{ Active } & \multicolumn{4}{|c|}{ Placebo } \\
\hline & \multicolumn{2}{|c|}{ First cycle } & \multicolumn{2}{|c|}{ Second cycle } & \multicolumn{2}{|c|}{ First cycle } & \multicolumn{2}{|c|}{ Second cycle } \\
\hline & Mean & SD & Mean & SD & Mean & SD & Mean & SD \\
\hline Genistein $(\mathrm{mg} / \mathrm{d})$ & $8 \cdot 78$ & $4 \cdot 97^{\star \star \star}$ & $7 \cdot 88$ & $3.97^{\star \star \star}$ & 0.52 & $0.73^{\star \star \star}$ & 0.67 & $0.98^{\star \star \star}$ \\
\hline Daidzein (mg/d) & $13 \cdot 87$ & $7 \cdot 64^{\star \star \star}$ & $12 \cdot 14$ & $4 \cdot 31^{\star \star \star}$ & 0.35 & $0.42^{\star \star \star}$ & 0.34 & $0.32^{\star \star \star}$ \\
\hline
\end{tabular}

${ }^{* \star \star} P<0.001$ (difference between active and placebo treatment).

$\dagger$ Reduced due to missing or incomplete $24 \mathrm{~h}$ urine data.

Table 6. Symptom change between screening and active treatments according to equol production* (Mean values with their standard deviations)

\begin{tabular}{|c|c|c|c|c|c|c|c|c|}
\hline \multirow[b]{3}{*}{ Symptom cluster } & \multicolumn{4}{|c|}{ Equol (mg/d) (n 6†) } & \multicolumn{4}{|c|}{ Non-equol (mg/d) (n 14 $)$} \\
\hline & \multicolumn{2}{|c|}{ Postmenstrual } & \multicolumn{2}{|c|}{ Premenstrual } & \multicolumn{2}{|c|}{ Postmenstrual } & \multicolumn{2}{|c|}{ Premenstrual } \\
\hline & Mean & SD & Mean & SD & Mean & SD & Mean & SD \\
\hline Total & -1.01 & 3.77 & -4.69 & $5 \cdot 20$ & -1.06 & 4.85 & -4.29 & $5 \cdot 73$ \\
\hline Mood & -0.02 & 1.60 & $-2 \cdot 61$ & 3.65 & -1.99 & 3.69 & -4.44 & $4 \cdot 19$ \\
\hline Behavioural & -0.88 & 0.76 & $-3 \cdot 17$ & 2.58 & -1.03 & 3.05 & -3.79 & 3.54 \\
\hline Pain & -0.28 & 0.41 & -1.52 & $1 \cdot 18$ & -0.38 & 0.88 & -1.93 & 1.66 \\
\hline Physical & -0.40 & 1.17 & -1.51 & 1.35 & -0.24 & 0.87 & -1.38 & 1.63 \\
\hline
\end{tabular}

*High negative value indicates greater symptom reduction.

†Reduced due to missing or incomplete $24 \mathrm{~h}$ urine data. 
effect was partly anticipated in the present study, although it was not possible to continue the study beyond two cycles per condition. In spite of the strict double-blind procedure and identical products, the response to placebo was substantial and was greater in the first cycle. Statistical analysis, which included both cycles and all cycle phases, did not find reductions in symptoms following soy IF that were significantly distinct from placebo. However, closer examination showed that the pattern of response between active and placebo treatments did differ. Whereas symptoms continued to fall in the second cycle of treatment after ISP containing IF, they were increased from the first to the second cycle of treatment with placebo, which suggests that the placebo effect in the current study was endured for at least 1 month. The exact period of time required to overcome a placebo response in relation to PMS is not well established. Some studies report non-specific effects for up to 4 months (Freeman \& Rickels, 1999). The separation of ISP containing IF and placebo, which was apparent in the current study, ISP containing might become clearer with further cycles of intervention and this should be examined in future studies. Focused statistical analysis on the second cycle of intervention to allow potential placebo effects to decline did provide suggestive evidence of a beneficial effect of soy IF on physical symptoms, although differences between active and placebo treatments remained non-significant. This could also indicate that the physiological effects of soy IF require two or more cycles of intervention for receptor sensitivity to increase rather than for placebo effects to decrease. Since there have been no studies that examine the biological effects of soy IF on symptoms across the menstrual cycle, this proposition requires investigation.

No clear mechanism has yet been proposed to explain how soy IF could reduce the symptoms of PMS. It can be postulated that the biological actions of soy IF reduce symptoms that are mediated by oestrogens or oestrogen receptors. Specifically, soy IF exert effects on oestrogen receptors and when endogenous concentrations are high (i.e. in premenopausal women) the weak oestrogenic nature of the soy IF results in anti-oestrogenic responses (Cassidy et al. 1994). Conversely, when endogenous oestrogens are limited (i.e. in postmenopausal women) soy IF appear to exert oestrogenic effects. These effects are also likely to occur in a similar fashion during the menstrual cycle, where the effect of soy IF will depend upon the circulating concentrations of oestrogen that fluctuate throughout the menstrual cycle (Cassidy et al. 1995; Hill \& Dye, 2003). Thus, ISP containing IF may exert an oestrogenic effect during the follicular phase (i.e. when oestrogen is low) and potentially an anti-oestrogenic effect in the luteal phase. In theory, soy IF may act to reduce premenstrual symptoms by stabilising natural cyclical fluctuations of oestrogen. Whilst hormonal profiles of women with PMS do not differ to those without PMS (Rubinow, 1992), interruption of the menstrual cycle (e.g. by ovariectomy) influences premenstrual symptoms and treatment with gonadotrophin-releasing hormone agonists that suppress ovulation can be effective in reducing PMS (Pearlstein $\&$ Steiner, 2000). These data support the theory that women with PMS react differently to normal cyclical fluctuations in sex hormones such as oestrogen via an as yet unknown mechanism purported to involve the hypothalamic-pituitary-gonadol axis, which influences neurotransmitter function in the central nervous system (Reid \& Yen, 1981; Ashby et al. 1988; Rubinow \& Schmidt, 1995; Walker, 1995; Mortola, 1996; Steiner et al. 1997; Korzekwa \& Steiner, 1999).
It is possible that soy IF effects are mediated through other non-classical mechanisms. For example, IF have a higher affinity for oestrogen $\beta$ receptors (Kuiper et al. 1996), which are found in the brain, bone, bladder and vascular epithelia (Cassidy \& Foughnan, 2000). It could be speculated that this relates more closely to reduction of specific symptoms. Alterations to cycle length with soy IF could have mediated symptom improvement. This has been demonstrated previously and relates to an elongation of the follicular phase only (Cassidy et al. 1994), although not all studies demonstrate cycle length alterations after soy consumption (Kurzer, 2002). Thus, increasing menstrual cycle length, specifically as a result of an increase in follicular phase length, may be beneficial in lowering symptoms as it reduces the overall exposure to oestrogen. Further studies using daily urine collections and symptom reporting are needed to confirm this.

Results of the present study were vulnerable to the influence of compliance, as most measures were made in a free-living environment. Participants were asked to consume ISP containing IF whilst avoiding all other foods containing phytoestrogens where possible. Analysis of urine samples collected during each intervention cycle revealed that levels of genistein and daidzein were increased only after active treatment, providing good evidence of compliance. However, completeness of urine collection could not be confirmed without a measure of $p$-aminobenzoic acid, although this is itself subject to interference and variation.

A high level of variation in genistein and daidzein was observed, due to individual variation in the metabolism of ISP containing IF, for instance, because of equol production. High variability in the excretion of soy IF metabolites is related mainly to gut microflora and dietary factors (Mallett \& Rowland, 1988; Wu et al. 2000; Setchell et al. 2002). Equol is a metabolite of ISP containing IF produced in the gastrointestinal tract by bacterial degradation of daidzein. Although evidence is not conclusive, variation is presumed to be caused by inter-individual differences in colonic bacteria (Setchell \& Cassidy, 1999). The clinical effectiveness of ISP containing IF may be dependent upon equol production, as it is believed to elicit greater biological reaction than other metabolites. In the present study, equol excretion did not differ between baseline and active treatments. This was anticipated, given the fact that up to $70 \%$ of the population are not able to produce notable amounts of equol (Xu et al. 1998). Amongst the twenty-three women in the present study, seventeen produced only trace amounts of equol and the influence of the six equol producers was not great enough to produce an overall change in equol after treatment. Moreover, comparisons between equol and non-equol producers revealed that symptom change was no different between these groups. It is presumed that this related to the small sample of equol producers in the study, although other unknown mechanisms cannot be ruled out. Thus, although this indicates that equol production may be unrelated to symptom change, this cannot be confirmed in the present study.

A further limitation of this study design relates to the high level of participant withdrawal during screening. The main reasons provided by participants were that the study protocol would be too demanding and the food supplement was disliked. Women were asked to record symptoms every day for a seven-cycle period, in addition to providing four $24 \mathrm{~h}$ urine samples. They were also asked to change their usual diets to accommodate the addition of soy products, and consumption of these produced aversive reaction in some participants. These issues were 
considered during protocol design, but a weaker between-subjects design for a shorter period was rejected, since this would require greater numbers of participants in each treatment condition and have lower power overall. The soy food product chosen had high quality control and confirmed reliable IF levels. The product was clinically produced according to good manufacturing practice and more reliable for research purposes than commercially available products. Whilst this enhanced quality control, it (and the extended duration of the study) resulted in inevitable losses of potential participants who disliked its taste or found the demands of the study too great. Future research should consider these limitations and improve clinical products to enhance its acceptability. The level of dropout experienced at an early stage in the study meant that intention to treat analysis could not be performed since post-randomisation loss meant that no primary endpoint measures were obtained on those women who failed to complete the study. It is also possible that the results were biased by the loss of participants, which may have disrupted the baseline equivalences established during the group assignment to treatment order. To account for this, baseline data are only reported for participants who fully completed the study protocol.

In conclusion, the present study has supported previous findings that ISP containing IF has some potential to treat premenstrual symptoms. Although total symptom severity was not improved significantly compared with placebo treatment, improvement of symptomatology related to somatic symptoms of swelling, headache, aches and breast tenderness was noted. It is unclear whether ISP containing IF exert a specific effect on somatic symptoms, or whether reductions in psychological symptoms are clouded by the strong placebo effect. Further research should therefore intervene for periods long enough to allow a return to baseline with reduced placebo effect. Additional studies are needed to corroborate these findings to determine the precise mechanism by which ISP containing IF exerts its actions and to assess the most effective dose and duration required.

\section{Acknowledgements}

M. Bryant acknowledges a scholarship grant from Leeds University. C. Hill was in receipt of a studentship from the Medical Research Council (grant reference: G27/7244). Unilever R\&D, Dr Jayne Ellis and Dr M. M. Gani at Unipath are thanked for their contribution in analysing the biochemical assays and The Solae Company for their generous donation of ISP containing IF and placebo products. The authors thank the volunteers for their cooperation during the study.

\section{References}

Ashby CR, Carr LA, Cook CL \& Steptoe MM (1988) Alteration of platelet serotonergic mechanisms and monoamine oxidase activity in premenstrual syndrome. Biol Psychiatry 24, 225-233.

Brienholt V \& Larsen JP (1998) Detection of weak estrogenic flavonoids using a recombinant yeast strain and a modified MCF7 cell profileration assay. Chem Res Toxicol 11, 622-629.

Bryant M \& Dye L (2004) Nutrient interventions for Premenstrual Syndrome (PMS): a review of the evidence for their efficacy. Curr Topics Nutraceutical Res 2, 201-219.

Burke BE, Olson RD \& Cusack BJ (2002) Randomized, controlled trial of phytoestrogen in the prophylactic treatment of menstrual migraine. Biomed Pharmacother 56, 283-288.
Cassidy A, Bingham S \& Setchell KD (1994) Biological effects of a diet of soy protein rich in isoflavones on the menstrual cycle of premenopausal women. Am J Clin Nutr 60, 333-340.

Cassidy A, Bingham S \& Setchell K (1995) Biological effects of isoflavones in young women: importance of the chemical composition of soyabean products. Br J Nutr 74, 587-601.

Cassidy A \& Foughnan M (2000) Phyto-oestrogens throughout the life cycle. Proc Nutr Soc 59, 489-496.

Cohen J \& Cohen P (1983). Applied Multiple Regression/Correlation Analysis for Behavioural Sciences. Hillsdale: Erlbaum.

Freeman E, DeRubeis R \& Rickels K (1996) Reliability and validity of a daily diary for premenstrual syndrome. Psychiatry Res 62, 97-106.

Freeman EW (2003) Premenstrual syndrome and premenstrual dysphoric disorder: definitions and diagnosis. Psychoneuroendocrinology 28, 25-37.

Freeman EW \& Rickels K (1999) Characteristics of placebo responses in medical treatment of premenstrual syndrome. Arch Gen Psychiatry 156, $1403-1408$.

Halbreich U (2003) The etiology, biology, and evolving pathology of premenstrual syndromes. Psychoneuroendocrinology 28, 55-99.

Hill CE \& Dye L (2003) Phytoestrogens and cognitive performance: a review of the evidence. Curr Topics Nutraceutical Res 1, 203-212.

Ingram DM, Hickling C, West L, Mahe LJ \& Dunbar PM (2002) A double-blind randomized controlled trial of isoflavones in the treatment of cyclical mastalgia. The Breast 11, 170-174.

Ishiwata N, Uesugi S, Uehara M \& Watanabe S (2005) Effects of soy isoflavones on premenstrual syndrome. Proceedings of the International Soy and Health Conference. Br J Nutr (In the Press).

Korzekwa MI \& Steiner M (1999) Assessment and treatment of premenstrual syndromes. Prim Care Update Ob Gyns 6, 153-162.

Kuiper GG, Enmark E, Pelto-Huikkp M, Nilsson S \& Gustafasson JA (1996) Cloning of a novel receptor expressed in rat prostate and ovary. Proc Natl Acad Sci USA 93, 5925-5930.

Kurzer MS (2002) Hormonal effects of soy in premenstrual women and men. $J$ Nutr 132, 570S-573S.

Lephart ED, Galindo E \& Bu LH (2003) Stress (hypothalamic-pituitaryadrenal axis) and pain response in male rats exposed lifelong to high vs. low phytoestrogen diets. Neurosci Lett 342, 65-68.

Lephart ED, West TW, Weber SW, Rhees R, Setchell K, Aldercreutz H \& Lund T (2001) Neurobehavioural effects of dietary soy phytoestrogens. Neurotoxicol Teratol 24, 5-16.

Mallett AK \& Rowland IR (1988) Factors affecting the gut microflora. In Role of the Gut Flora in Toxicity and Cancer, pp. 347-382 [IR Rowland, editor]. London: Academic Press.

Maskarinec G, Franke AA, Williams AE, Hebshi S, Oshiro C, Murphy S \& Stanczyk FZ (2004) Effects of a 2-year randomized soy intervention on sex hormone in premenstrual women. Cancer Epidemiol Biomarkers Prev 13, 1736-1744.

McFadyen IJ, Chetty U, Setchell KDR, Zimmer-Nechemias L, Stanley E \& Miller WR (2000) A randomized double blind-cross over trial of soya protein for the treatment of cyclical breast pain. Breast $\mathbf{9}$, 271-276.

Mortola JF (1992) Issues in the diagnosis and research of premenstrual syndrome. Clin Obstet Gyn 35, 587-597.

Mortola JF (1996) Premenstrual Syndrome. Trends Endocrinol Metab 7, 184-189.

Muse KN, Cetel NS, Futterman LA \& Yen SSC (1984) The premenstrual syndrome: effects of 'medical ovariectomy'. N Engl J Med 311, $1345-1349$.

National Institute of Mental Health (1983) NIMH Premenstrual Syndrome Workshop Guidelines. Rockville, MD: National Institute of Mental Health.

Pearlstein T \& Steiner M (2000) Non-antidepressant treatment of premenstrual syndrome. J Clin Psychiatry 61, 22-27.

Reid RL \& Yen SSC (1981) The effect of [beta]-endorphin on arginineinduced growth hormone and prolactin release. Life Sci 29, 2641-2647. 
Rowland IR, Faughnan M, Hoey L, Wahala K, Williamson G \& Cassidy A (2003) Bioavailability of phyto-oestrogens. Br J Nutr 89, S34-S58. Rowland IR, Wiseman H, Sanders TA, Adlercreutz H \& Bowey EA (2000) Interindividual variation in metabolism of soy isoflavones and lignans: influence of habitual diet on equol production by the gut microflora. Nutr Cancer 36, 27-32.

Rubinow DR (1992) The Premenstrual Syndrome: new views. J Am Med Assoc 268, 1908-1912.

Rubinow DR \& Schmidt PJ (1995) The treatment of Premenstrual Syndrome - forward into the past. N Engl J Med 332, 1574-1575.

Schnurr PP (1989) Measuring amount of symptom change in the diagnosis of premenstrual syndrome. J Consult Clin Psychol 1, 227-283.

Setchell K, Brown N \& Lydeking-Olsen (2002) The clinical importance of the metabolite equol - a clue to the effectiveness of soy and its isoflavones. J Nutr 132, 3577-3584.

Setchell K \& Cassidy A (1999) Dietary isoflavones: biological effects and relevance to human health. $J$ Nutr 129, 758-767.

Setchell KD, Borriello SP, Hulme P, Kirk DN \& Axelson M (1984) Nonsteroidal estrogens of dietary origin: possible roles in hormone-dependent disease. Am J Clin Nutr 40, 569-578.
Singh B, Berman B, Simpson R \& Annechild A (1998) Incidence of premenstrual syndrome and remedy usage: a national probability sample study. Altern Ther Health Med 4, 75-79.

Steiner M, Coote M, Wilkins A \& Dunn E (1997) Biological correlates of irritability in women with premenstrual dysphoria. Euro Neuropsychopharmacol 7, S172.

Tabachnick BG \& Fidell LS (1996) Using Multivariate Statistics, 3rd ed. New York: Harper Collins.

VanStrien T, Frijters J, Bergers G \& Defares P (1986) The Dutch Eating Behavior Questionnaire (DEBQ) for assessment of restrained, emotional and external eating behavior. Int J Eat Disord 5, 295-315.

Walker A (1995) Theory and methodology in premenstrual syndrome research. Soc Sci Med 41, 793-800.

Wu AH, Stanczyk FZ, Hendrich S, Murphy PA, Zhang C, Wan P \& Pike MC (2000) Effects of soy foods on ovarian function in premenopausal women. Br J Cancer 82, 1879-1886.

Xu X, Duncan AM, Merz BE \& Kurzer MS (1998) Effects of soy isoflavones on estrogen and phytoestrogen metabolism in premenopausal women. Cancer Epidemiol Biomarkers Prev 7, 1101-1108. 